This item was submitted to Loughborough's Research Repository by the author.

Items in Figshare are protected by copyright, with all rights reserved, unless otherwise indicated.

\title{
Tutorial on means of hybrid simulation
}

PLEASE CITE THE PUBLISHED VERSION

https://doi.org/10.1109/WSC40007.2019.9004712

PUBLISHER

IEEE

VERSION

AM (Accepted Manuscript)

PUBLISHER STATEMENT

(c) 2019 IEEE. Personal use of this material is permitted. Permission from IEEE must be obtained for all other uses, in any current or future media, including reprinting/republishing this material for advertising or promotional purposes, creating new collective works, for resale or redistribution to servers or lists, or reuse of any copyrighted component of this work in other works.

\section{LICENCE}

All Rights Reserved

REPOSITORY RECORD

Eldabi, Tillal, Antuela Tako, David Bell, and Andreas Tolk. 2020. "Tutorial on Means of Hybrid Simulation". figshare. https://hdl.handle.net/2134/12159177.v1. 


\title{
TUTORIAL ON MEANS OF HYBRID SIMULATION
}

\author{
Tillal Eldabi \\ Surrey Business School \\ University of Surrey \\ Guildford \\ Surrey GU2 7XH, UK
}

David Bell

Department of Computer Science

Brunel University London

Wilfred Brown Building

Uxbridge, Middx UB8 3PH, UK

\author{
Antuela A. Tako \\ School of Business and Economics \\ Loughborough University \\ Loughborough \\ Leicestershire, LE11 3TU, UK \\ Andreas Tolk \\ The MITRE Corporation \\ 903 Enterprise Parkway \#200 \\ Hampton, VA 23666, USA
}

\begin{abstract}
The benefits of Hybrid Simulation (HS) are well recorded in academic literature. It offers deeper insights into the real-life system as it allows modelers to assess its inherent problems from different dimensions. As a result HS has recently generated more attention from within the Modeling and Simulation arena. HS comes in many shapes and forms. For example, by linking two or more simulation models; linking simulation models with facilitative models; or linking simulation models with analytical models. This paper aims to explore several concepts related to HS modelling and design.
\end{abstract}

\section{INTRODUCTION}

It has been argued by many researchers that hybrid simulation provides a better insight of the system in hand as it allows modelers to assess its inherent problems from different dimensions (Zulkepli and Eldabi 2015). The need for HS is predominantly fueled by the ever rising complexity of the modern world and the rise of information sources and data. This led Hybrid Simulation to become an important subject within the Modeling and Simulation field. There are several types of Hybrid Simulations (for example, hybrid models that are based on two or more simulation models such as linking discrete event simulation (DES) with either system dynamics (SD) or agent based simulation (ABS) models). Another example is linking simulation models with facilitative or analytical models from the wider Operations Research domain or disciplines such as Systems Engineering and Applied Computing (Powell and Mustafee 2017). HS started life as a process of pragmatic mixing of several paradigms/approaches/techniques, making it difficult to be housed within one specific clear methodology (Zulkepli and Eldabi 2015). Tolk et al. (2018) attempted to establish some categorization of HS by extending the classification originally proposed by Mustafee et al. (2017). That initiative classifies hybridization of different models with respect to their approaches (traditionally used interchangeably with techniques in the simulation community) as listed below:

- When techniques from different simulation methodologies are combined, such as ABS and SD, this is called multi-methodology hybrid simulation; 
- When techniques from the same simulation methodologies are combined, such as SD and (continuous) computational fluid dynamics CFD models, this is called multi-technique hybrid simulation;

- When different techniques from the same methodology and different simulation methodologies are combined, the result is a multi-methodology- multi-technique hybrid simulation;

- Combining simulation methods with non-simulation methods, as originally proposed in (Shanthikumar and Sargent 1983), is called a multi-methodology hybrid model;

- Combing methods from different paradigms is called a multi-paradigm hybrid model.

The above classification is too complex for the purpose of this paper. To simplify things we divide the HS approaches into two categories: Hybrid Simulation i.e. mixing/linking one simulation model (ABS, DES, or SD) with one or more other simulation model(s). The second category is Hybrid Modelling: which is mixing between one or more simulation model(s) (ABS, DES, or SD) and other modelling approach(es). This is stemmed from the fact that when discussing HS, we are mainly focusing on the process of simulation and the approaches followed to design develop and utilize a simulation model. In this paper we will explore some issues related to designing and developing HS starting by a proposed framework for hybrid model development, followed by facilitative approaches to HS, the use of HS to resolve complex issues, concluding by defining some theories for linking disparate paradigms with particular emphasis on linking the physical and simulation paradigms.

\section{A PROPOSED HS FRAMEWORK}

This framework is based on three sequential phases: the conceptual phase; the modeling phase; and the model communication phase. The following sections provide detailed description of the three stages.

\subsection{Phase 1: Conceptual Phase}

Step One: Problem Source Definition and Objective(s) Identification. This is a typical step that is vital and performed for simulation models to define the problem and the need for hybridization.

Step Two: 'Conceptual Model and Modularization Process'. This step aims to subdivide the conceptual model into several modules, each of the modules contains a standalone process. Taking a healthcare example each of the modules could be a model of a hospital clinic, social care, A\&E, surgery, etc.). It may also subdivide the same model into further dimensions for example a patient model and a doctor model. The main purpose of modularization is to simplify complex structures and drawing the boundaries for the various hybridized models at the conceptual phase.

Step Three: Identification of the Characteristics of each Module. The purpose of this step is to identify the characteristics of each module to decide which technique is suitable for modeling the respective module. Such characteristics are: short- (or long) term effect; type of analysis (individual or aggregate), feedback requirement etc. Modelers could resort to well-established characteristics used to define the three main simulation approaches i.e. DES, SD, or ABS. The identification of the characteristics and requirements for each module will lead to deciding which approach to follow.

\subsection{Phase 2: Modeling Phase}

The second phase in the framework is the modeling phase. Each of the models (i.e. DES, SD, ABS) can be developed separately and simultaneously if expertise is available. The development of each of the models should be based on the previous phase. These models can be developed using normal commercial and freely available packages. The most important aspect in this phase that is different from a typical simulation development phase is the facilitation of the linking between the models. 


\subsection{Phase 3: Models Communication Phase}

Phase 3 of the framework is about defining the linking and development of a communication plan between the different models. The purpose of this phase is about identifying how variables from some models communicate and change their impact on other variables in other model. The 'communication' process also includes how each of the models are changing accordingly. The linkage between models is decided by identifying the 'influencing' and the 'influenced' variables that make these models communicate with each other. The following discussion provides the explanation of the six steps involved in this phase.

Step One: Identifying the Linking Variables. The identification of such variables is done by detecting all the variables that 'influence' or 'are influenced' in both models. Expert opinion can be sought to validate which variables can be considered as 'influence' variables and which are 'influenced' variables. It is worth noting that the techniques used to model these variables may also depend on the modelers' expertise.

Step Two: Identifying the Interactions Type. Chahal and Eldabi (2008) introduced two types of interaction, or model communication, cyclic interaction and parallel interaction. Cyclic interaction is based on running one module to generate input and the next module. Parallel interaction is about contentiously and mutually transferring data between the models during the run.

Step Three: Model Execution and Data Exchange. The 'variables involved' are those identified in Step One as 'influence' or 'influenced' variables. As each model may have an initial influencing variable, we will use the 'source model' as the general term in this framework to define the model with the initial 'influencing' variable(s), whilst the 'destination model' contains the initial 'influenced' variable(s).

Step Four: Evaluation of Outputs. The final output can be from either the 'source' or the 'destination' model. That is the final output will be from the destination model unless that there are some variables in the source model which are influenced by the variables in the destination mode. In such case the final output will be traced from the destination model.

\section{FACILITATIVE HYBRID SIMULATION}

From its definition hybrid simulation involves the modelling of complex systems, which are best represented using one or more simulation or non-simulation methods combined together. As a result, this creates the need for capturing different perspectives and sources of information to inform the different parts of the model or stages of the modelling lifecycle. This section considers the potential of adopting facilitated modelling in the lifecycle of hybrid simulation modelling.

The concept of facilitated modelling is not new, as it has been applied in a number of operational research techniques, where a subjective analysis of the problem (e.g. many views incorporated) is carried out and a model is built with the view to identifying desirable and feasible solutions (Franco and Montibeller, 2010). The process involves developing models jointly with a client group, face-to-face, with or without the assistance of computer support (Eden and Radford, 1990, Franco and Montibeller, 2010). It is particularly beneficial when modeling systems with complex behavior, involving many stakeholders with plurality of opinions and objectives.

Facilitated modeling has been applied separately in the three most popular methods combined in hybrid simulation, including: discrete-event simulation (Robinson et al. 2014, Tako and Kotiadis 2015, Proudlove et al. 2017, Kotiadis and Tako 2018), system dynamics (Andersen and Richardson, 1997, Rouwette et al., 2000, Vennix, 1999, Lane et al. 2019) and agent-based modelling (Chu et al. 2012). While their definitions and focus may differ, a common element is the aspect of developing models in a participatory approach, with the involvement of a client group at different stages of the simulation study. The extent and maturity of the facilitation practice also differs across the different methods. We next consider, "How can the existing practice in facilitated simulation modelling help hybrid modelling?"

By its nature hybrid modelling is particularly useful for modelling complex problems. Methods from different paradigms and approaches are combined to provide a representation of a system at different levels. Due to the complexity of the system and the different levels of abstraction required, facilitated modelling 
can prove useful in achieving an agreement between the different stakeholder groups involved and to achieve common modelling objectives. The stakeholder groups involved include the following:

- The modelers. These could be researchers with the different modelling skills coming from different modelling paradigms and disciplines. Hybrid simulation is particularly useful for modelling complex problems that are better solved by involving expertise from various disciplines and communities (Mustafee et al. 2017).

- The client. Members from different parts of the organization or system, responsible to operate and represent different parts of the system modeled. They know the system well and understand how it operates, even though not necessarily as a whole.

Through facilitated modelling these two stakeholder groups can come together to develop commonly agreed models at the conceptual phase (phase 1), which are then used to evaluate different options and scenarios at the model communication phase (phase 3 ). The timely engagement of a group of stakeholders in the study provides the opportunity to mould the study to their collective requirements that can also instil a sense of ownership of the study and its outcomes.

Involving different stakeholder groups from multiple research fields and communities requires a process of elicitation of views that can be achieved through facilitated modelling. This requires a very welldesigned process consisting of group activities that allow for the participation and involvement of all stakeholders in sessions led by a facilitator. This process is particularly useful for reaching agreed problem definitions and conceptual models, based on which the combined models are developed. The facilitation process aims to support the extraction of information relevant to developing a representative CM and at the same time encouraging creativity through divergent and convergent thinking (Tako and Kotiadis 2012). Currently there is no specific conceptual modelling approach available to support hybrid simulation. There are however methods such as for example qualitative system dynamics (Powel and Mustafee 2017, Vennix 1996) and the PartiSim framework that can be adapted to support the knowledge elicitation process in the conceptual phases of the hybrid modelling lifecycle. Further work is however needed to design facilitated activities to involve a group of stakeholders in identifying appropriate links between the different submodels developed, which is particularly relevant to the current needs of hybrid simulation.

During the model coding stage, facilitated sessions involving the client are not recommended. This is also the case in single-method facilitated simulation, where the models are built in the back office (Robinson et al. 2014). Particularly for hybrid simulation this stage is not compatible to the client group participation due to the technical complexity involved. Facilitation could however be useful in the case when multiple modelers are involved. Facilitated sessions with the modelers can prove helpful in understanding the links between their models, especially in cases where modelers from multi or inter-disciplinary communities are involved (Mustafee et al. 2017).

After the model is developed, facilitated workshops can reconvene in the models communication phase, where the two stakeholder groups engage in group activities that explore the solution space (equivalent to experimenting with the model). This is further narrowed down to feasible and desirable action to be taken by the client organization to identify an implementation plan. Kotiadis and Tako (2018) provide detailed activities and tools that can be used by the facilitator and the stakeholder group, which can be also adapted to hybrid simulation. On the other hand, Scriptapedia (Scriptapedia, 2012) is also available, which provides an online platform where modelers can share GMB scripts for use in facilitated system dynamics (Andersen and Richardson 1997; Hovmand et al. 2012). The main attribute of the facilitation process followed is that it provides a forum where model results are discussed between the modeler and stakeholder group and subsequent actions to be taken are co-produced (Kotiadis and Tako, 2018).

To conclude, facilitated simulation encourages plurality of opinions and the achievement of consensus among the stakeholders groups involved. The process enables the individual stakeholders to express their individual viewpoints and agendas freely at workshops, so that the group can reach commonly agreed models. It requires group processes, including open discussions, reflection points, voting if necessary, etc 
that make it possible for a group of stakeholders to be involved. Therefore, any conflicting opinions arising can be resolved within the workshops. Both the process and the resulting outputs are transparent to the group of stakeholders involved.

\section{ON DEVELOPING HS FOR A TYPICAL EXAMPLE OF COMPLEX SYSTEMS (DAVID BELL)}

\subsection{Context}

Healthcare has been a popular home for hybrid simulation (Gao et al. 2014; Onggo 2014; Viana 2014; Fakhimi et al. 2015, Raghothama et al. 2017 ), often providing opportunities to explore wider system issues. Literature has typically focused on case studies, clearly demonstrating practical viability of the approaches. Fewer publications however have explored the modelling processes (including conceptual modelling), especially when designing across organizational boundaries in a data-driven manner. The project presented here was initiated in London to explore several strategic changes being made to the wider healthcare care system. A system dynamics model was first developed to simulate the impact of both the expected changes in baseline demand (demographics) as well as the identified strategic service changes on the performance of the system. This focused specifically on the demand for unplanned care. Applying the strategic service changes resulted in changes to the simulated level of demand for unplanned care. The variation in demand was simulated over time in the model outputs. This provided the basis for selecting alternative sets of input data to the operational model, based on: 1) Strategic service change that were in play or planned, 2) estimated impact and timing of each strategic service change and 3) time points at which the resulting demand was modelled.

\subsection{Traditional DES Modelling}

SIMUL8 software was used to build a DES for the emergency care pathway. The simulation mapped patient activity for one year through urgent care services. It used transactional data from the Health and Social Care Information Centre (http://www.hscic.gov.uk/) to accurately build a baseline of flow through these different services. Data was segregated by patient age, arrival method and arrival time to build distributions of arrival, routing and length of stay for patients in different services, including (i) discharge by hour, (ii) length of stay by hour and (iii) paediatric arrival. Separate distributions and routing patterns were tested against baseline data to ensure accuracy. Once an accurate baseline was established pre-defined changes were tested in the software, these changes explored the impact of different patient numbers using alternative services. The fine-grained segmentation of data enabled testing of changes more precisely, especially when looking at specific demographic and strategic impacts from SD modelling.
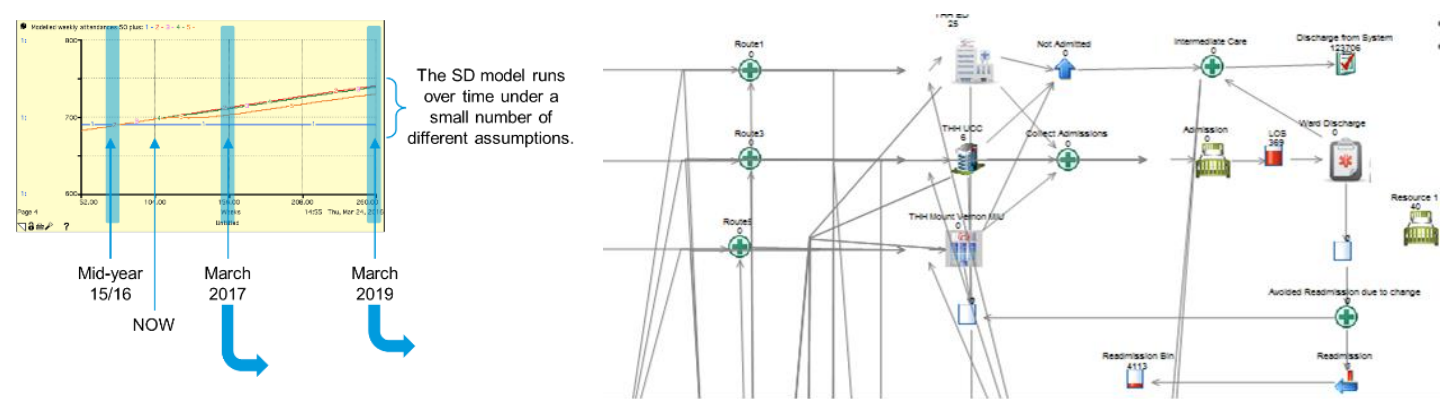

Figure 1 SD strategic output directing a DES model (Bell et al. 2016). 
Traditional approaches where used to build the two models in Figure 1. Stakeholder interviews with service experts were carried out to understand the services and planned strategic change. Findings were then presented to senior managers within the regional management team. In parallel, interviews were carried out with expert users of the transactional data (approximately 130,000 records in the extract used for this research). Subsequent analysis of the data enabled the modelers to produce the DES models with aforementioned distributions. The connectivity between models was chosen to reflect the times of resulting stable impact from the strategic changes taking place (after a suitable length of time to bed in). Hybridization was undertaken using software engineering approaches and coupling code was developed accordingly. Unsurprising, with two teams building their respective models, conceptual and semantic differences were apparent.

\subsection{Post-hoc Conceptual Modelling}

After completing the hybrid simulation experiments, a further iteration was carried out in order to find suitable semantic integration tools and techniques that could hopefully reduce the conceptual differences and ambiguities encountered. Formal concept analysis (FCA) was chosen and used to analyze instance data (the same transaction data used by the DES modeler) in order to uncover categories and relationships. Figure 2 presents this analysis using the FCART tool.

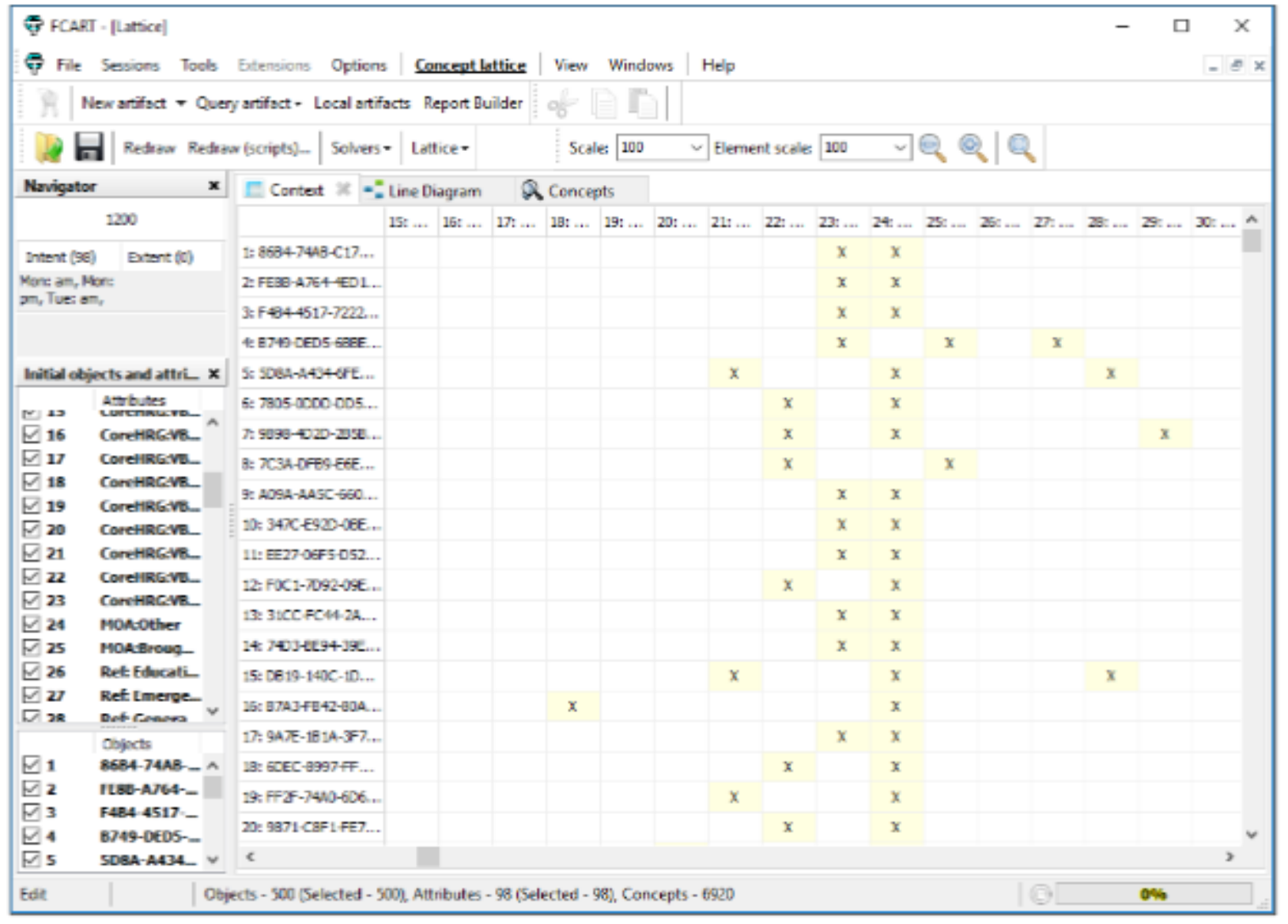

Figure 2 Formal Concept Analysis. 
After completing the FCA, concepts were used to extend the emergency care pathways using space-timeevent maps to include the new conceptual language (uncovering temporal parts of a pathway - Sider 2003 - and associated events). The basic structures of FCA are context and concept hierarchy. A context in this care being comprised of a set of Patient IDs as objects, a set of attributes, and a binary relation describing which objects include which attributes.

\section{HS/CPS PROSPECTS AND EXAMPLE (ANDREAS TOLK)}

Cyber physical systems (CPS) are generally defined as a new generation of systems with integrated computational and physical capabilities that can interact with humans through many new modalities (Baheti and Gill 2011). Obviously, simulation plays an important role regarding the computational capabilities. These multi-modalities and the many application domains of CPS result in the use of a multitude of different modeling paradigms and heterogeneous solutions. Even if the CPS community is not identifying this composition of heterogenous simulation solutions as hybrid simulation, it de facto is one of the fastest growing application domains for hybrid simulation solutions, and as such provides an excellent use case to identify some general challenges. These challenges can be summarized in the task to provide a common foundation for supporting homogenous solutions based on the heterogenous applications of hybrid simulation approaches. The correlated topic is well known in the CPS community, but it nonetheless requires more research and attention: how to mix the continuous control elements of the physical world with digital twins or valid co-simulation representations in the computational domain? Both challenges will be addressed in the following subsections.

\subsection{Homogeneous Solutions despite Heterogeneous Applications}

George Bernhard Shaw is usually attributed with the quote that "the English and Americans are two peoples divided by a common language." The same can be said for the applicants of hybrid modelling and simulation approaches from different scientific disciplines. This underlying problem has been described in a recent study in which Chen and Crilly (2016) evaluated commonality of issues between practitioners in the field of synthetic biology and swarm robotics and showed that these practitioners shared more complexity related issues between each other than they did with colleagues in their original domain. Nonetheless, the sharing of information and reuse of solutions was hindered by the different terms and concepts used to describe them within their home domain. CPS are used in many different domains. They contribute to the Internet of Things as much as to the Industry 4.0 efforts. CPS are parts of smart cities, smart houses, smarter cars, and more. And although they may use the same methods and paradigms to address a problem, they may not be able to know it, as the language and concepts of the hosting disciplines are simply too different, as the example of Chen and Crilly demonstrates. Furthermore, in an expert discussion during the Spring Simulation Multi-Conference 2018 (Tolk et al. 2018), the need for a common formalism to express the computational capabilities with focus on hybrid simulation was addressed by several of the participating experts. Hybrid simulation addresses more possible combination, as exemplified by Traore's contribution to Tolk et al. (2018) that is captured in the following figure.

The various formalisms shown in this figure are dealing with discrete solutions, continuous simulations, operational research solutions, and functionality provided by physical capabilities of devices. It shows applicable methods on the levels of conceptualization, specification, and implementation can be combined in CPS solutions, which in addition have to be tagged with terms provided in the commonly accepted glossary.

We are therefore faced with a multitude of terms and definitions applicable in various contexts of a huge diversity of formalisms and modeling and simulation approaches. A common glossary of terms, maybe even a common ontology being able to express more logical constraints and connections, will be needed in addition to tap a common formal representation. Furthermore, the clear definition of the formalisms used is needed to be captured. The resulting recommendations for the user of hybrid modelling and simulations can be summarized as follows: 


\begin{tabular}{|c|c|c|c|c|}
\hline $\begin{array}{c}\text { Concepts } \\
\text { (formalisms) }\end{array}$ & $\begin{array}{l}\text { DEVS, Petri } \\
\text { Net, Multi- } \\
\text { Agents... }\end{array}$ & $\begin{array}{c}\text { ODE, PDE, } \\
\text { System } \\
\text { Dynamics... }\end{array}$ & $\begin{array}{l}\text { OR methods, AI } \\
\text { methods... }\end{array}$ & \\
\hline $\begin{array}{c}\text { Specifications } \\
\text { (models) }\end{array}$ & $\begin{array}{l}\text { Discrete } \\
\text { simulation } \\
\text { models } \\
(\text { DisM) }\end{array}$ & $\begin{array}{l}\text { Continuous } \\
\text { simulation } \\
\text { models } \\
(\text { ContM })\end{array}$ & $\begin{array}{l}\text { Algorithms } \\
\text { (Alg) }\end{array}$ & \\
\hline $\begin{array}{c}\text { Operations } \\
\text { (engines) }\end{array}$ & Simulators' & Integrators & $\begin{array}{l}\text { Solvers' } \\
\text { (Sol) }\end{array}$ & $\begin{array}{l}\text { Physical device } \\
\text { (Phy) }\end{array}$ \\
\hline & $=\frac{M S}{.6}$ & onal worlo & $\overline{\mathrm{W}})$ & \\
\hline
\end{tabular}

DEVS:Discrete Event System Specification PDE: Partial Differential Equations

AI: $\quad$ Artificial Intelligence

----: often referred to as combined simulation
ODE: Ordinary Differential Equations

OR: Operation Research

----: often referred to as hybrid simulation

----: CPS

Figure 4 Spectrum of Hybrid Simulation in CPS.

1. Create at least a clear glossary that defines the terms used in your study. Each terms used to tag an entity, a property, a process, an attribute, or a state needs to be defined in this glossary. If possible, additional logical constraints needs to be captured, preferably in machine readable form, which leads to an ontology.

2. The used formalisms to capture concepts, specifications, and operations for discrete, continuous, operations research, and physical capabilities need to be documented in detail.

3. The interconnection and mapping points of these formalism need to be documented with particular detail to support validation of such compositions.

Theoretical mathematics provide several good approaches. The use of model theory has already been proven to be valuable in evaluating the consistent representation of truth as a requirement for composability in heterogenous modeling environments (Diallo et al. 2014) and is likely to be applicable as a unifying approach supporting validation of hybrid approaches as well. The second subsection presents more specific contribution of mathematics, hybrid automata.

\subsection{Integrating Continuous Physical Elements and Digital Computational Elements}

Lee (2006) proposed the use of hybrid automata to represent CPS for simulation-based approaches, as they combine finite state machines with ordinary differential equations (ODE), both powerful concepts used in physics modeling. Extending the ideas of Henzinger (1996) and Alur et al. (2000), these hybrid automata have non-deterministic finite states, where each states can be described by an ODE. The work originally was developed as a mathematical model to address digital computational process within continuous environments. As such, Lee was able to show their applicability to represent the computational and physical capabilities of CPS accordingly, providing a common mathematical foundation.

A hybrid automata can best be described as a directed graph in which the vertices or nodes are called control states, and the edges are the switches between the control states. Each status has three predicates that work on the variables describing the discrete and continuous values of interest. Overall, up to $n$ variables are observed, with $n$ also being called the dimension of the automaton, formally written as a vector $X=\left[x_{1}, x_{2}, \ldots, x_{\mathrm{n}}\right]$. In some publications, the discrete variables and continuous variables are captured in separate vectors, as the continuous variables need to be solved differently, namely using ODE. The three predicates capture the initial, invariant, and flow conditions with free variables from all variables with the 
exception of the flow condition which only uses the continuous variables. The events that trigger the change of states are based on the predicates of the nodes they are connecting, which is often referred as the jump condition of states. This formalism allows to describe the state a CPS is in, and which are possible following states, using a common representation. The following figure shows a thermostat as an example.

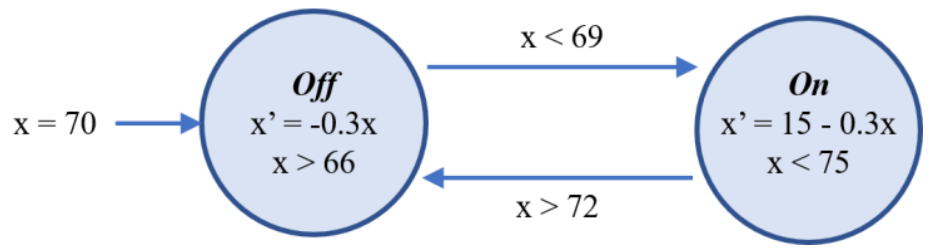

Figure 5 Example of a Heater.

In this example, the only variable is the temperature of the room. Initially, it is 70 degrees. The heater control can be Off or On, and initially it is off. The jump conditions to change from off to on is a temperature under 69 degrees. The jump condition from on to off is a temperature above 72 degrees. The first line in the nodes describes the flow condition to trigger the event, how fast the temperature changes, the second the invariant condition, the current temperature itself. The change from on to off is triggered when the temperature rises too quickly, or when it is hotter than 75 degrees.

This simple example demonstrates the main ideas and already allows to construct more complex system representations. Henzinger (1996) introduced many additional ideas, including the use of labels, compositions, feedback loops, all contributing to a powerful formalism that is understandable for humans, but also machine readable. Only recently, these ideas were modified and extended by Mustafiz et al. (2016) in their work on the modular design of hybrid languages. They do not only address the need for support of discrete and continuous methods, they explicitly mention the general challenge of encompassing multiple levels of abstraction and combining different formalisms, often not expressible in any single existing formalisms. They also address the challenge that the different supporting communities may use different terms to address the same phenomenon, or use the same term to describe different phenomena in their domains. Therefore, they conclude that the creation of new hybrid languages specifically tailored for such supported domains involves not only composition of the syntax but also the semantics. They introduce the idea of Hybrid Timed Finite State Automata (TSFA) - extending the ideas of hybrid automata - and used causal block diagrams for the synchronization and orchestration of process execution. This combination of formalisms from discrete and continuous approaches on various abstraction levels shall ultimately allow the description of ever more complex CPS and allow their simulation in a consistent and comparable form, using the approaches of the CPS community as the basis for their hybrid languages.

In summary of this section, CPS are a growing application field for hybrid modeling and simulation. As already observed by Mustafiz et al. (2016), the reason for such interest is that "the engineering of a complex CPS involves the creation and simulation of hybrid models often encompassing multiple levels of abstraction and combining different formalisms, often not expressible in any single existing formalisms." In this section, we addressed the need for a hybrid formalism able to include methods from discrete, continuous, operations research, and physical aspects of a CPS on the conceptual level to allow for the consistent implementation of all contribution capability specification. This requires not only a common formal approach, but also a common glossary unambiguously defining all terms used to interpret the formal representation. As discussed in Mustafee et al. (2017), "hybrid M\&S results from using two or more components of different $M \& S$ categories to generate something new, that combines the characteristics of these components into something more useful for the underlying $M \& S$ effort to be supported that are composable under the constraints of this effort." CPS are an application domain that require this new approach, as they are literally composed of the many domains addressed by hybrid efforts being the topic of this tutorial. The interested scholar may refer to Mittal and Tolk (2019), which provides an in-depth study of the topic of hybrid M\&S, complexity, and CPS. 
Eldabi, Bell, Tako, and Tolk

\section{CONCLUSIONS}

This paper focusses on Hybrid Simulation as a rising topic with many potentials and yet many challenges. The paper attempts to tackle four elements within the grand Hybrid Simulation domain in order to contribute to the practice of Hybrid Simulation by providing some guiding steps. The first element proposes a high level framework for developing simulation models, in terms of deciding whether to hybridize and some guiding steps to follow. The second element looks at facilitative Hybrid Simulation as a process that encourages plurality of opinions and the achievement of consensus among the stakeholders groups involved. The process enables the individual stakeholders to express their individual viewpoints and agendas freely at workshops, so that the group can reach commonly agreed models. It requires group processes, including open discussions, reflection points, voting if necessary, etc that make it possible for a group of stakeholders to be involved. Therefore, any conflicting opinions arising can be resolved within the workshops. Both the process and the resulting outputs are transparent to the group of stakeholders involved. The third element provides an effective method for conceptual model validation, prior to distinct streams of Hybrid Simulation model building and coupling. When comparing the conceptual language between the earlier project teams and this later semantic analysis, a substantial number of new concepts were uncovered. The fourth element looks at a fast rising category that goes beyond the realm of modelling as a cyber space and presents theories related to developing links between the physical world and the cyber models.

\section{ACKNOWLEDGEMENTS}

The authors would like to thank Nurul Saleh for her Formal Concept Analysis technical work that supported this tutorial.

\section{REFERENCES}

Alur, R., T. A. Henzinger, G. Lafferriere, and G. J. Pappas. 2000. "Discrete Abstractions of Hybrid Systems". Proceedings of the IEEE 88(7): 971-984.

Andersen, D. F. and G.P. Richardson. 1997. "Scripts for Group Model Building”. System Dynamics Review 13(2): $107-129$.

Baheti, R. and H. Gill. 2011. "Cyber-Physical Systems". The Impact of Control Technology 12(1): 161-166.

Chahal, K. and Eldabi, T. 2008. "Applicability of Hybrid Simulation to Different Modes of Governance in UK Healthcare". In Proceedings of the 2008 Winter simulation conference, December $7^{\text {th }}-10^{\text {th }}$, Miami, USA $1478-1483$

Chen, C. C. and N. Crilly, N. 2016. "Describing Complex Design Practices with a Cross-Domain Framework: Learning from Synthetic Biology and Swarm Robotics". Research in Engineering Design 27 (3): 291-305.

Chu, T. Q., A. Drogoul, A. Boucher, and J. D. Jucker. 2012. "Towards a Methodology for the Participatory Design of Agent-Based Models" In Principles and Practice of Multi-Agent Systems, Edited by, N. Desai, A. Liu, M. Winikoff. Lecture Notes in Computer Science, vol 7057. Springer, Berlin, Heidelberg.

Diallo, S. Y., J. J. Padilla, R. Gore, H. Herencia-Zapana, and A. Tolk. 2014. "Toward A Formalism of Modeling and Simulation using Model Theory". Complexity 19(3): 56-63.

Eden, C. and K. J. Radford. 1990. Tackling Strategic Problems: The Role of Group Decision Support, London: Sage Publications,.

Fakhimi, M., L.K. Stergioulas, N. Mustafee. 2015. "An Investigation of Hybrid Simulation for Modeling Sustainability in Healthcare". In Proceedings of the 2015 Winter Simulation Conference, December $6^{\text {th }}-9^{\text {th }}$, Huntington Beach, USA 15851596.

Franco, L.A. and G. Montibeller. 2010. "Facilitated Modelling in Operational Research." European Journal of Operational Research 205(3): 489-500.

Gao, A., N. D., N. Osgood, W. An, R. F. Dyck. 2014. "A Tripartite Hybrid Model Architecture for Investigating Health and Cost Impacts and Intervention Tradeoffs for Diabetic End-Stage Renal Disease". In Proceedings of the 2014 Winter Simulation Conference, December $7^{\text {th }}-10^{\text {th }}$, Savannah, USA.

Henzinger, T. 1996. "The Theory of Hybrid Automata". In Proceedings of the 11th Annual IEEE Symposium on Logic in Computer Science, July $27^{\text {th }}-30^{\text {th }}$, New Jersey, USA 278-292.

Hovmand, P. S., D. F. Andersen, E. A. J. A. Rouwette, G. P. Richardson, K. Rux, and A. Calhoun. 2012. "Group Model-Building 'Scripts' as a Collaborative Planning Tool”. Systems Research and Behavioral Science, 29(2): 179-193.

Kotiadis, K. and A. A. Tako. 2018. "Facilitated Post-Model Coding in Discrete Event Simulation (DES): A Case Study in Healthcare". European Journal of Operational Research, 266 (3): 1120-1133.

Lane, D., E. Husemann, D. Holland, A. Khaled. 2019. "Understanding Foodborne Transmission Mechanisms for Norovirus: a Study for the UK's Food Standards Agency”. European Journal of Operational Research, 275(2): 721-736. 
Eldabi, Bell, Tako, and Tolk

Lee, E. A. .2006. “Cyber-Physical Systems-Are Computing Foundations Adequate?". In NSF Workshop On Cyber-Physical Systems: Research Motivation, Techniques and Roadmap (Vol. 2); National Science Foundation: Washington, DC.

Mittal, S., and A. Tolk. 2019. Complexity Challenges in Cyber Physical Systems: Using Modeling and Simulation (M\&S) to Support Intelligence, Adaptation and Autonomy NJ: John Wiley and Sons, Inc.

Mustafee, N., Brailsford, S., Djanatliev, A., Eldabi, T., Kunc, M. and A. Tolk. 2017. "Purpose And Benefits of Hybrid Simulation: Contributing to the Convergence of its Definition." In proceedings of the 2017 Winter Simulation Conference. December $3^{\text {rd }}$ - 6th Las Vegas, USA 1637-1645.

Mustafiz, S., C. Gomes, B. Barroca, and H. Vangheluwe. 2016. "Modular Design Of Hybrid Languages by Explicit Modeling of Semantic Adaptation". In Proceedings of the Symposium on Theory of Modeling \& Simulation, April $3^{\text {rd }}-6^{\text {th }}$ Pasadena, USA 29-36.

Onggo, B. S. 2014. "Elements of a Hybrid Simulation Model: A Case Study of the Blood Supply Chain in Low- and Middleincome Countries". In Proceedings of the 2014 Winter Simulation Conference, December $7^{\text {th }}-10^{\text {th }}$, Savannah, USA 15971607.

Powell, J. H. and N. Mustafee. 2017.” Widening Requirements Capture with Soft Methods: an Investigation of Hybrid M\&S Studies In Health Care”. Journal of the Operational Research Society 68(10): 1211.

Proudlove, N. C., S. Bisogno, B. S. S. Onggo, A. Calabrese, and N. Levialdi Ghiron. 2017. "Towards Fully-Facilitated Discrete Event Simulation Modelling: Addressing the Model Coding Stage". European Journal of Operational Research, 263(2): 583595.

Raghothama, J., H. Hanchi and S. Meijer. 2017. "Hybrid, Composable Approach to Simulations in Healthcare Operations and Management". In proceedings of the 2017 Winter Simulation Conference, December $3^{\text {rd }}-6^{\text {th }}$ Las Vegas, 4449-4449.

Robinson, S., C. Worthington, N. Burgess, and Z. J. Radnor. 2014. "Facilitated Modelling with Discrete-Event Simulation: Reality or Myth?". European Journal of Operational Research, 234(1): 231-240.

Rouwette, E. A. J. A., J. A. M. Vennix, and C. M. Thijssen. 2000. “Group Model Building: a Decision Room Approach”. Simulation And Gaming, 31(3): 359-379.

Scriptapedia, 2012. In Wikibooks. https://en.wikibooks.org/wiki/Scriptapedia, accessed 14.5.2019.

Sider, T. 2001. Four-Dimensionalism: An ontology of Persistence and Time”. Oxford University Press on Demand.

Tako A. A. and K. Kotiadis. 2015. "PartiSim: A Framework for Participative Simulation Modelling". European Journal of Operational Research, 244 (2): 555-564.

Tako, A. A., and K. Kotiadis. 2012. "Facilitated Conceptual Modelling: Practical Issues and Reflections". In Proceedings of the 2012 Winter Simulation Conference December $9^{\text {th }}-12^{\text {th }}$, Berlin, Germany 1-12.

Tolk, A., S. Mittal, and E. H. Page. 2018. "Hybrid Simulation: Frameworks, Methods, and Foundations with Focus on Cyber Physical Systems." MITRE Technical Report MTR180341, McLean, Virginia: The MITRE Corporation.

Tolk, A., F., A. Barros, A. D’Ambrogio, P. J. Rajhans, S. S. Mosterman, M. K. Shetty, H. Traore, L. Vangheluwe,and L. Yilmaz. 2018. "Hybrid Simulation for Cyber Physical Systems: A Panel on Where Are We Going Regarding Complexity, Intelligence, and Adaptability of CPS Using Simulation”. In Proceedings of the Symposium on M\&S of Complexity in Intelligent, Adaptive and Autonomous Systems. April $15 \mathrm{t}-18^{\text {th }}$, Baltimore, USA.

Vennix, J. A. M. 1996. Group Model-building: Facilitating Team Learning Using System Dynamics. Chichester, Wiley.

Viana, J. 2014. "Reflections on Two Approaches to Hybrid Simulation in Healthcare". In Proceedings of the 2014 Winter Simulation Conference, December $7^{\text {th }}-10^{\text {th }}$, Savannah, USA 1585-1596.

Zulkepli, J., and T. Eldabi. 2015. "Towards a Framework for Conceptual Model Hybridization in Healthcare". In Proceedings of the 2015 Winter Simulation Conference, December $6^{\text {th }}-9^{\text {th }}$, Huntington Beach, USA 1597-1608.

\section{AUTHOR BIOGRAPHIES}

TILLAL ELDABI is a senior lecturer at University of Surrey. He has B.Sc.in Econometrics and M.Sc. and Ph.D. in Simulation Modeling in Healthcare. His research is into aspects of healthcare modeling and simulation. He developed a models support health economists and clinicians to decide on best treatment programs. He has published widely in the field of modeling in healthcare and edited a number of special issues in related journals. His email address is t.eldabi@surrey.ac.uk, and his home page is https://www.surrey.ac.uk/people/tillal-eldabi .

ANTUELA A. TAKO is is a Reader in Operational Research at the School of Business and Economics, Loughborough University. She holds a PhD in Simulation and an MSc in Management Science and Operational Research from the University of Warwick. Her research interests include the comparison of simulation approaches (discrete-event simulation and system dynamics), facilitated and participative simulation modelling, conceptual modelling and health care modelling. She is Associate Editor of the Journal of the Operational Research Society and of the Journal of Simulation. Her email address is a.a.takou@lboro.ac.uk and her webpage is http://www.lboro.ac.uk/departments/sbe/staff/anthi-antuela-tako/.

DAVID BELL is a Reader in Computer Science at Brunel University London. He also holds a PhD in Computer Science from 
Brunel University London. His research interests include data-driven M\&S, cyber security and Web semantics. His email address is david.bell@brunel.ac.uk

ANDREAS TOLK is Senior Principal at The MITRE Corp. His affiliation with The MITRE Corporation is provided for identification purposes only. He is an adjunct Full Professor at Old Dominion University, a senior member of ACM and IEEE, and a Fellow of the Society for Modeling and Simulation (SCS). His email is atolk@ mitre.org. His OrcId is 0000-0002-4201-8757. His contributions have been approved for Public Release, Distribution Unlimited, Case Number 18-2167-18. 